\title{
TWENTY YEARS FORWARD
}

\section{COL (RET) PAUL F. PASQUINA, MD; CHAIRMAN OF PHYSICAL MEDICINE AND REHABILITATION, UNIFORMED SERVICES UNIVERSITY OF THE HEALTH SCIENCES}

\section{INTRODUCTION}

\ $t$ is very humbling to provide commentary to JRRD's 50th anniversary edition, especially in light of the enormous contributions that the Department of Veterans Affairs (VA) and Department of Defense (DOD) have made in the advancement of rehabilitative medicine over the past half century.

Since the Civil War, the United States and its citizens have been inspired by the words of President Lincoln, who during his second inaugural address on March 4, 1865 stated-

With malice toward none, with charity for all, with firmness in the right as God gives us to see the right, let us strive on to finish the work we are in, to bind up the nation's wounds, to care for him who shall have borne the battle and for his widow, and his orphan, to do all which may achieve and cherish a just and lasting peace among ourselves and with all nations.

In response to President Lincoln's call to action, countless individuals have dedicated their lives and professional careers to caring for our nation's servicemembers and families. The same holds true today. During the last decade of war, I have had the privilege of serving in various capacities within the military healthcare system, most recently as the Chief of Orthopaedics and Rehabilitation at Walter Reed National Military Medical Center in Bethesda, Maryland. These positions have afforded me the unique opportunity to meet and work with some of the most dedicated and exceptional professionals within the VA and DOD healthcare systems. I am continually amazed at not only their expertise, but also their tireless efforts to apply their skills to improve the lives of our servicemembers and veterans and their families. Moreover, they demonstrate relentless determination and intellectual curiosity toward the discovery of new therapeutic interventions to further improve the care available to our nation's heroes. Their work is inspiring and infectious to healthcare professionals, scientists, and researchers across the globe.

As history has shown us, optimal care for combat casualties requires coordinated interdisciplinary care by professionals across medical, surgical, rehabilitative, and psychological health disciplines. Providing coordinated care, however, presents its own unique challenges, especially in today's healthcare environment where many physicians, therapists, nurses, psychologists, social workers, orthotists, and prosthetists have become subspecialized to the point where they risk losing sight of the patient's overall well-being. While the advancements in each of these specialties offer improved diagnostic capabilities and enhanced treatment options, we must remain aware of the dynamic between specialties to ensure that each treatment plan is coordinated to best achieve the individual patient's goals.

As scientific discoveries continue to surface, teamwork will play an increasingly vital role in the advancement of care for combat casualties. Advances in fields such as robotics, regenerative medicine, genetics, genomics, and neuroscience have the potential to ignite a synergy across disciplines in order to revolutionize tomorrow's healthcare. In addition, evidence continues to emerge indicating the benefits of recreational activities, sports, peer support, and the creative arts on rehabilitation, recovery, resilience, and reintegration after injury or illness-further emphasizing the expanding contributions of various disciplines to healthcare advances. Determining the optimal coordination of these disciplines will require leadership within the DOD and VA healthcare systems to build a model for the country to follow. Furthermore, we must ensure that the lessons learned from the past decade of war, especially regarding the positive effect of interdisciplinary and

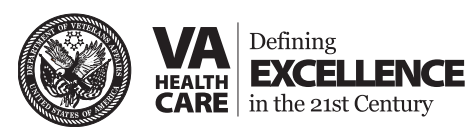


xviii

JRRD, Volume 50, Number 10, 2013

multidimensional care, are not lost to future generations of military and veteran healthcare providers. Therefore, our educational systems must not only carry forward these lessons learned, but also be able to adapt to healthcare advances and rapidly integrate new discoveries into military-unique curricula to ensure these discoveries are expeditiously disseminated throughout clinical care settings.

In reference to the 1964 issues of JRRD (then known as the Bulletin of Prosthetics Research), it is clear that many of the observations made nearly 50 years ago still remain true today and will undoubtedly remain true for future generations of military healthcare providers. Four elements stand out in the care of servicemembers and veterans with limb loss: (1) the powerful effect and high visibility that servicemembers with amputation continue to have on the American public, (2) the importance of strong partnership between the DOD and VA systems in providing care for combat casualties, (3) the positive influence that congressional leadership can have on improving the lives of combat casualties and veterans, and (4) the importance of DOD and VA directed research and fostering public/private partnerships. These elements are discussed in turn in the subsequent sections.

Powerful Effect and High Visibility that Servicemembers with Amputation Continue to Have on American Public

While a relatively small percentage of combat casualties from Operations Iraqi Freedom and Enduring Freedom have sustained a major limb amputation (estimated to be approximately 1,600 as of June 2013), the images remain indelible in the public's eye. Perhaps it is the symbolic nature of a servicemember's sacrifice of a "part of him- or herself" in the service of this nation or the countless examples of the resilience that veterans with limb loss have displayed since the Civil War in achieving great personal and professional success despite their physical impairment. Their cause has the ability to unite us as a nation, and their advocacy has had a powerful effect on healthcare policy and public awareness. Moreover, their leadership, whether in uniform, political office, industry, or academia, has had a tremendous effect on improving access to better assistive technology, education, employment, sports, and recreational activities for all individuals with disabilities within the United States and abroad.

Importance of Strong Partnership Between DOD and VA Systems in Providing Care for Combat Casualties

The DOD-VA partnership has become critical for servicemembers with blast casualties, who frequently sustain multiple coexisting injuries such as limb loss, traumatic brain injury, paralysis, vision/hearing impairment, and/or psychological problems. Many of these patients require extensive medical and surgical interventions extending over months or years postinjury, but are still able to remain on Active Duty. Therefore, their ability to receive seamless care across the DOD and VA systems expands the capacity of both healthcare systems to provide state-ofthe-art care to more patients across a greater geographical area within the continental United States. Furthermore, evidence demonstrates the importance of initiating rehabilitation for these patients as soon as possible during their acute medical and surgical care. Delaying rehabilitation until the combat casualty's acute medical and surgical issues are resolved and he or she is able to be transferred to the VA system leads to an increase in complications and has devastating repercussions on recovery. Therefore, a system of care that is well coordinated between the DOD and VA and sharing expertise in caring for combat casualties are critical for success.

Interestingly, considerable debate persists regarding overlapping roles of the DOD and VA systems in caring for individuals with limb loss. Experience over the past decade clearly demonstrates the need to maintain amputee care programs in both healthcare systems. Each program, however, should have the capacity to expand or contract to be commensurate with the volume and complexity of patients seen or anticipated during a time of war, national disaster, or humanitarian efforts abroad. It is also important to remember that the vast majority of individuals with limb loss seen in the VA system sustain amputation from diseases such as vascular insufficiency or diabetes and not the traumatic wounds that are most commonly seen in the DOD system. Ensuring expertise in both systems fosters better collaboration, sharing of experiences, and ultimately improved care for our servicemembers and veterans and their families.

Positive Influence that Congressional Leadership Can Have on Improving Lives of Combat Casualties and Veterans

While political philosophies may differ throughout the United States, during times of war, political leaders have consistently united to support veterans and combat casualties. With each war, federally funded programs have consistently contributed to incremental improvements in the care 
and well-being of servicemembers with disabilities. Many of these programs have also greatly contributed to improved healthcare for the general public. This is particularly true in trauma and rehabilitative care: programs to support the development and reimbursement of advanced prosthetic components, sensory aids, and assistive technology, as well as those that create educational and vocational opportunities for veterans with disabilities, have greatly improved the independence, quality of life, and productivity of injured servicemembers. These programs not only help define us as nation that respects human life and treasures its heroes, but also help to strengthen our military forces, as many servicemembers join the military with the full faith and confidence that if they are injured while serving in harm's way they will receive only the best of care.

\section{Importance of DOD and VA Directed Research and Fostering Public/ Private Partnerships}

Over the past half-century, research studies across a breadth of disciplines have enhanced our knowledge of the challenges facing combat casualties to include improved outcome measures to assess domains such as independent living, mobility, mental health conditions, substance abuse, and quality of life.

These advances have informed the treatment of combat casualties from initial injury to long-term care. Perhaps the clearest demonstration of the advancement of combat casualty care even over the past decade is the significant improvement in survival rates of patients with combat wounds. This has been attributed to enhanced body armor, advanced resuscitative trauma care, and improved medical evaluation techniques. Similarly, recent decades have brought significant improvements in our ability to diagnose and treat conditions such as wound infections, blood clotting, heterotopic bone formation, pain syndromes, traumatic brain injury, and posttraumatic stress disorder. With respect to some of the advances that have been made in caring for individuals with extremity injuries, including amputation, surgical subspecialists are now able to preserve limbs and limb length that previously were unsalvageable.

These improvements in acute casualty care have been paralleled by advances in rehabilitation. Researchers have elucidated the biomechanical influences on the human body with various prosthetic components and mobility devices, such as manual and motorized wheelchairs, to help improve independent function and reduce long-term overuse injuries to remaining upper- and lowerlimb joints. Superior prosthetic materials have improved socket fit and comfort, improving prosthetic wear time and reducing skin breakdown. Newly designed dynamic response feet allow more natural ankle and foot function for various activities, including less energy loss, better vertical shock absorption, rotation, and even ankle motion as needed. Advanced prosthetic knees now have built-in microprocessors that respond to forces within the prosthetic limb to adjust knee resistance at fractions of a second to allow variable cadence walking on various terrains. In addition, novel, commercially available powered knee and ankle systems for the first time restore energy and power to ambulation, substituting for lost muscles.
These powered devices may allow faster self-selected walking speeds and ease ascending inclines or stairs. Furthermore, these devices may ameliorate the increased risk of long-term medical and musculoskeletal conditions faced by veterans with physical disabilities, reducing the occurrence of secondary complications such as arthritis and back pain. In summary, there have been many advances in the care of combat casualties over just the past decade, many of which would not have been possible without the direct support and leadership of the DOD and VA research programs.

Thanks to continued DOD and VA efforts, future discoveries are also on the horizon. Current studies suggest that further discoveries in regenerative medicine, robotics, neuroscience and brain mapping, enhanced by advanced neurological and body imaging techniques (e.g., magnetic resonance imaging [MRI], functional MRI, single-photon emission computed tomography, positron emission tomography, diffuse tensor imaging, high-resolution ultrasound), and biological markers (e.g., genetics, genomics, proteomics, cytokines) will improve diagnostic and treatment options for combat casualties, particularly those with multitrauma, extremity injuries, amputation, traumatic brain injury, paralysis, and mental health problems. Improved humanmachine interface strategies, such as the direct skeletal attachment of a prosthesis through osseointegration or improved control through a brain-machine interface, and/ or implantable microstimulators or microelectrode sensors, have the potential to revolutionize prosthetic use, providing both improved motor control as well as haptic sensation. 
Finally, improved powered/motorized lower-limb systems (prosthetics or exoskeletons) will likely allow individuals with lower-limb loss and/ or paralysis enhance mobility in a manner that replicates near-normal biomechanics, with the secondary positive effect of reducing long-term health risks (e.g., cardiovascular disease, diabetes, bone health, arthritis, overuse injury).

\section{CONCLUSIONS}

Despite the remarkable accomplishments in combat casualty and veteran care that have marked the past 50 years, there remains much to be done to improve the lives of the servicemembers, veterans, and families who have paid this tremendous sacrifice while serving their country. We are forever indebted to their service and must always remain committed to providing not only life-long care, but also improved quality of care that is informed by new discoveries across all disciplines. 